\title{
Search for $\mu-e$ conversion with DeeMe experiment at J-PARC MLF
}

\author{
N. M. Truong*, on behalf of DeeMe Collaboration \\ Osaka University, Toyonaka, Osaka, Japan \\ E-mail: truongekuno-q.phys.sci.osaka-u.ac.jp
}

DeeMe project is an experiment searching for muon to electron conversion $(\mu-e$ conversion) signal with single event sensitivity at level $10^{-14}$ by using a pulsed proton beam from J-PARC Rapid Cycling Synchrotron (RCS). The $\mu-e$ conversion is a process called charged-Lepton Flavor Violation. This process is essentially forbidden in the Standard Model of particle physics, therefore, this is one of clean processes to study physics beyond the Standard Model.

DeeMe experiment is proposed at J-PARC Materials and Life Science Experimental Facility (MLF) and it will be conducted at a new beam line (H-Line). The pulsed proton beam from RCS with 3-GeV energy and $25 \mathrm{~Hz}$ repetition is used to bombard a production target. DeeMe experiment utilizes muonic atoms produced inside the production target itself as a source of electrons for the $\mu-e$ conversion process. The electron emitted from the production target will be transported to a magnet spectrometer by $\mathrm{H}$-Line. A single event sensitivity is estimated to be $2 \times 10^{-14}$ with silicon-carbide-target and $1 \times 10^{-13}$ with graphite-target, providing $1-2$ orders of magnitudes improvements over the current upper limits $\left(4.3 \times 10^{-12}\right.$ for Ti and $7 \times 10^{-13}$ for Au). Details of the experimental technique, beam line preparation and their expected performance are presented.

Flavor Physics \& CP Violation 2015,

May 25-29, 2015

Nagoya, Japan

\footnotetext{
*Speaker.
} 


\section{Introduction}

When a negative muon enters matter, it will slow down, knock out electrons in one of the atoms and quickly form a muonic atom by orbiting single nucleus. After that, two phenomena will happen in the framework of the Standard Model (SM) of particle physics: muon decay in orbit (DIO) process, $\mu^{-} \rightarrow e^{-} v_{\mu} \overline{v_{e}}$, and muon capture (MC) process, $\mu^{-}+(A, Z) \rightarrow v_{\mu}+(A, Z-1)$. In addition, one more phenomenon may happen in nuclear field, $\mu^{-}+(A, Z) \rightarrow e^{-}+(A, Z)$; this phenomenon is called $\mu-e$ conversion process.

The $\mu-e$ conversion process in nuclear field is one of charged-lepton flavor violating (cLFV) processes, in which lepton number of each generation is not conserved. Based on the prediction of SM with the extension for neutrino oscillation, the branching ratio of the cLFV process is $\sim 10^{-50}$ [U, ㅁ]. This braching ratio level is out of any experimental technologies today, so that in the SM of particle physics point of view, cLFV processes is still forbidden substantially. On the other hand, a numerous theoretical models beyond SM predict that the cLFV processes will happen at level of few orders of magnitudes below upper limits given by the previous experiments and the $\mu-e$ conversion process is considered as one of the most powerful probes to search for the physics beyond SM [3], 因, []]. Any experiments that observe $\mu-e$ conversion signal will give clear evidence for new physics beyond the SM.

There have been many experiments in the world try to observe $\mu-e$ conversion signal, however this phenomenon has not been observed yet. The current upper limits of the branching ratio for the $\mu-e$ conversion process in nuclear field are given by the SINDRUM-II experiment at PSI, 4.3 $\times 10^{-12}$ for titanium target [四] and $7 \times 10^{-13}$ for gold target $[\square]$. These experimental results are close with the theoretical predictions of some physics beyond the SM, thus there are great potential for experiments to find $\mu-e$ conversion signal if we improve our sensitivity only a few orders of magnitudes. Even if we do not find any signal of the $\mu-e$ conversion process, the update of the upper limit on the branching ratio will help us improve the knowledge about physics beyond the SM.

\section{DeeMe experiment}

DeeMe experiment is an experiment searching for the $\mu-e$ conversion in nuclear field [ [ $]$ ]. The experiment will be conducted at H-Line of J-PARC MLF. A RCS will provide 3-GeV proton beam to MLF. The proton beam from RCS has double pulse structure, $200 \mathrm{~ns}$ width for each pulse, $600 \mathrm{~ns}$ sepration between two pulses, and $25 \mathrm{~Hz}$ repetition frequency. Figure 1 shows a concept of DeeMe experiment. DeeMe searches the $\mu-e$ conversion process with following procedure:

- proton beam hits the production target and produces pions;

- pions decay to muons inside the target;

- muons are captured by nuclei and muonic atoms are formed;

- muonic atoms emit electrons out of the target;

- H-Line is used to transfer electrons to the magnet spectrometer;

- the electrons' momenta are measured by using the magnet spectrometer. 


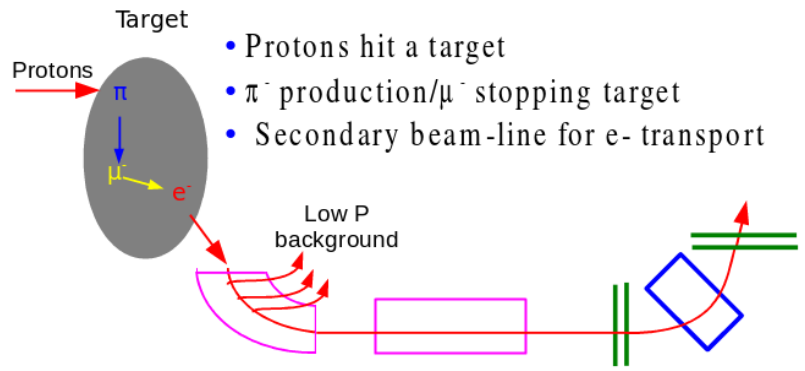

Figure 1: A concept of DeeMe experiment

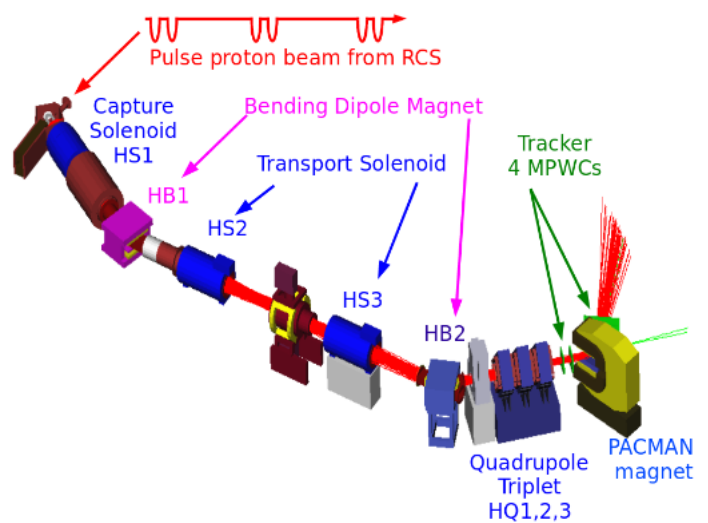

a) Layout of $\mathrm{H}$-Line in by G4BEAMLINE simulation

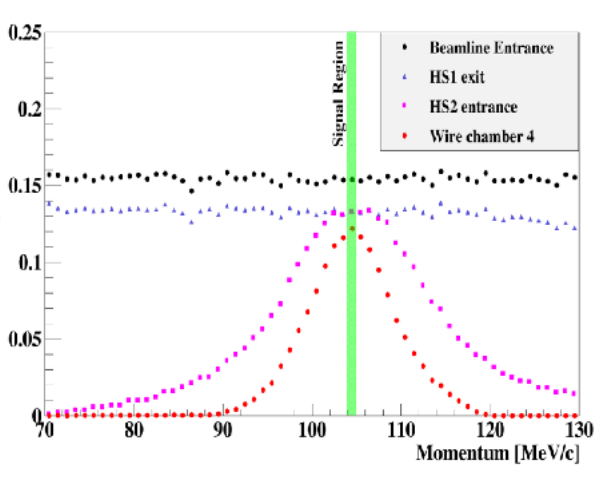

b) Acceptance of H-Line at components of the beam line as a function of the momentum

Figure 2: a) Layout of H-Line and the magnet spectrometer b) beamline acceptance of H-Line

H-Line, which are being constructed in MLF, is the general beam line for many different types of fundamental muon physics experiments such as MuSIUM [Q] g-2/EDM [ए0], etc. In DeeMe experiment, $\mathrm{H}$-Line is used to transfer electrons with momenta from $90 \mathrm{MeV} / c$ to $120 \mathrm{MeV} / c$. Low energy particles and positive charge particles will be removed during the transportation. Figure $2 \mathrm{a}$ shows a layout of H-Line and our magnet spectrometer. The magnet spectrometer, which includes a rectangular dipole magnet and four trackers, is placed at the end of the beamline to measure momenta of transported electrons. A Monte Carlo simulation study of the beam optics was done to optimize the beamline performance for momenta of the signal electrons from $\mu-e$ conversion (105 MeV/c) by using G4BEAMLINE package [ए]]. Figure $2 b$ shows the acceptance of H-Line and the magnet spectrometer as a function of momentum. Only electrons which have momenta from $90 \mathrm{MeV} / c$ to $120 \mathrm{MeV} / c$ can reach the magnet spectrometer.

\section{Background Control}

In DeeMe experiment, there are four kinds of major backgrounds which can mix with signal: prompt background, DIO background, after-proton background (APB) and cosmic rays background. 
Prompt background is huge amount of negative charged particles produced simultaneously when proton beam hits the production target. The momenta of these particles can reach to the momentum of $\mu-e$ conversion signal, $105 \mathrm{MeV} / c$, and they can mix with $\mu-e$ conversion signal in the signal region. However, this phenomenon only happens when the experiment is performed with continuous beam. On the other hand, DeeMe experiment uses the pulsed beam and observes only delayed electrons, thus we can remove these prompt backgrounds by using data in delayed-time region.

DIO electrons are Michel electrons that come from the decay of $\mu^{-}$in $1 S$ orbit of muonic atoms. Thus, this background has the same timing with $\mu-e$ conversion signal. Because of the recoil effect of the nucleus, the end point of DIO electrons' momenta is extended to the momentum of $\mu-e$ conversion electrons. However, the falling edge of the DIO momentum spectrum at its end-point scales with $\left(E_{\mu-e}-E_{e}\right)^{5}$, where $E_{\mu-e}$ is $\mu-e$ conversion signal energy and $E_{e}$ is electron energy at end-point of DIO spectrum [ए2]. Therefore, DIO electrons background can be suppressed by raising the lower energy threshold in the magnet spectrometer.

APB may be produced by the primary protons hitting the production target at the delayed timing. The proton beam which we use to bombard the production target is fast extracted proton beam from J-PARC RCS, therefore, it should not have any protons at delayed timing in principle. However, the APB can be induced by beam halo since they can be extracted to MLF by multiple scattering though the extraction kicker of RCS is off. These protons could induce the background in delayed timing, it will mix with signal region. Based on the data which we measured at RCS in 2014, the ratio of the number of after-protons to the total protons in RCS is less than $2 \times 10^{-18}$, it leads to APB reached to the magnet spectrometer less than 0.04 hits per year. This means that the APB is really small. Moreover, in order to estimate how much APB will affect to our real data, we will record the activities of the magnet spectrometer system before the extraction of RCS. Figure 3a shows more clearly how we mornitor the APB in real data taking.

Cosmic rays can hit the production target and induce electrons which have energy close to $\mu-e$ conversion signal. These electrons can be transferred to the magnet spectrometer system by $\mathrm{H}$-Line. These signal can mix with $\mu-e$ conversion signal in analysis region. DeeMe experiment suppresses this background by using two major techniques. First of all, as we can see in Figure 2a, the magnet spectrometer system of DeeMe experiment is placed in horizontal orientation, this will help to suppress cosmic ray backgrounds compare with others experiment such as SINDRUMII experiment. The second one is small live-time duty factor of detector in DeeMe experiment. The primary proton beam is $25 \mathrm{~Hz}$, our time window for analysis is $2 \mu \mathrm{s}$, thus the duty factor is $\sim 1 / 20000$. This helps to suppress cosmic ray backgrounds strongly. Furthermore, in the real data taking, we use self-trigger to readout the cosmic rays backgrounds during beam-off period. This technique permits to monitor cosmic rays backgrounds in very long time: 10000 times longer than the observing time of $\mu-e$ conversion.

\section{Single Event Sensitivity}

The single event sensitivity (SES) is expressed as 


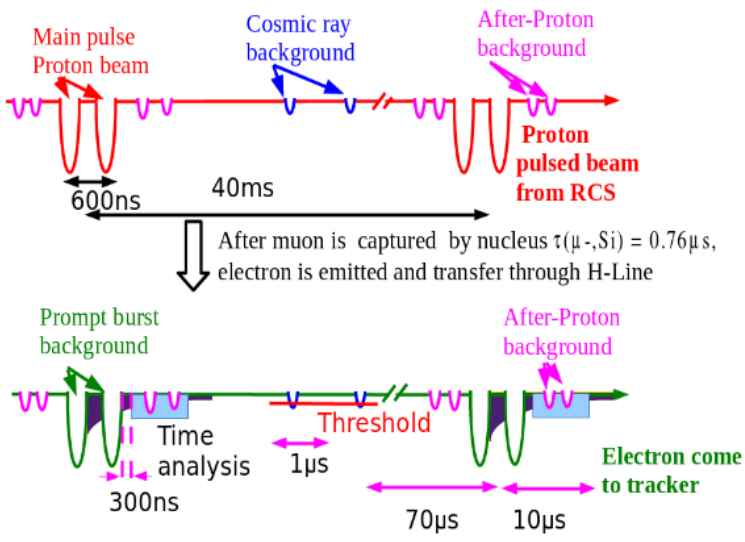

a) Background control

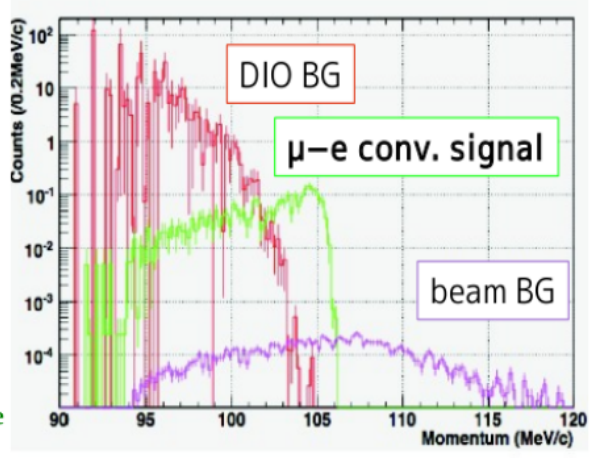

b) Momentum spectra of muon to eletron coming from muon to electron conversion, DIO and APB

Figure 3: Background control and momentum spectra of electrons

$$
S E S=\frac{1}{R_{\pi^{-}} \times f_{\pi^{-} \rightarrow \mu^{-} s t o p} \times f_{C} \times f_{M C} \times A_{\mu-e} \times T},
$$

where $R_{\pi^{-}}$is a $\pi^{-}$production rate, $f_{\pi^{-} \rightarrow \mu^{-}}$stop is a fraction of stopping muons in the production target, $f_{C}$ is the fraction of atomic capture of muons on the atom of interest, $f_{M C}$ is the fraction of muon nuclear capture, $A_{\mu-e}$ is the total acceptance for electrons from $\mu-e$ conversion. The simulated momentum spectra of electron from the $\mu-e$ conversion process, DIO, and APB for the silicon-carbide-target are shown in Figure 3b. In DeeMe experiment, we will use 3-GeV 1-MW proton beam for one year data taking $\left(2 \times 10^{7} \mathrm{~s}\right)$, the SES expected is $2 \times 10^{-14}$ for silicon-carbidetarget target and $1 \times 10^{-13}$ for graphite-target. Because of using proton beam from RCS, DeeMe experiment does not interfere with any experiments using a $30-\mathrm{GeV}$ main ring at J-PARC. We will have chance to reach the SES of $5 \times 10^{-15}$ if we run the experiment for 4 years.

\section{Summary}

DeeMe project is an experiment searching for the $\mu-e$ conversion in nuclear field. It will be conducted at J-PARC MLF. High power and high purity 3-GeV pulsed proton beam from RCS is used to produce muonic atoms in the production target. We aim to achieve the SES at level of $10^{-14}$ for one year data taking with silicon-carbide-target and it is posible to extend the SES down to level of $10^{-15}$ with 4 year data taking.

\section{References}

[1] S. M. Bilenky, S. T. Petcov, and B. Pontecorvo, Phys. Lett. B 67, 309 (1977).

[2] S. T. Petcov, Yad. Fiz. 25, 641 (1977) [Sov. J. Nucl. Phys. 25, 340 (1977)].

[3] E. J. Chun, K. Y. Lee, S. C. Park; N. Kakizaki, Y. Ogura, F. Shima, Phys. Lett. B 566, 142 (2003) 
[4] V. Cirigliano, A. Kurylov, M. J. Ramsey-Musolf, P. Vogel, Phys. Rev. D70, 075007 (2004)

[5] M. Blanke et al., JHEP 05013 (2007)

[6] C. Dohmen et al., Phys. Lett. B317, 631 (1993).

[7] W. Bertl et al., Eur. Phys. J. C 47, 337 (2006).

[8] M. Aoki, AIP Conference Proceedings 1441, 599 (2012).

[9] K. Shimomura et al., AIP Conf. Proc. 1382 (2011) 245

[10] H. Iinuma, J. Phys. Conf. Ser. 295 (2011) 012032

[11] http://www.muonsinternal.com/muons3/G4beamline

[12] O. Shanker, Phys. Rev. D 251847 (1982). 